\title{
Utilizing Electron Beam Control and Radiation Chemistry during Liquid Cell Electron Microscopy to Image Protein Aggregates in their Native Hydrated State
}

\author{
Taylor J. Woehl ${ }^{1}$ \\ ${ }^{1 .}$ University of Maryland, College Park, Department of Chemical and Biomolecular Engineering, \\ College Park, USA.
}

Protein aggregates are agglomerates of individual protein monomers, bound either through irreversible strong interparticle interactions or weak reversible interactions. Protein aggregates are implicated in the pathogenesis of several neurological disorders and are the main mechanism by which biopharmaceuticals, i.e. protein-based drugs, lose their stability during production, storage, and shipping $[1,2]$. The structure of protein aggregates varies widely depending on the protein type and the conditions under which the aggregate was formed and determines how they interact with biological systems. Conventional methods for determining protein structure include ensemble spectroscopy methods like NMR and x-ray scattering, and direct imaging approaches like negative-stain and cryo transmission electron microscopy (TEM). However, due to their electron beam sensitive nature and heterogeneous size and structure, none of these approaches are capable of determining the true structure of individual protein aggregates in their native hydrated state.

We are currently working on in situ liquid cell electron microscopy (LCEM) methods for imaging protein aggregates in their near-native hydrated state with nanometer scale spatial resolution [3]. While this approach allows for direct imaging of nanoscale samples in a thin $(\approx 100 \mathrm{~mm})$ liquid layer [4], radiation damage quickly deteriorates the tertiary and quaternary structure of protein aggregates $[5,6]$. Radiation damage is due mainly to oxidizing radicals created from electron beam induced radiolysis in the liquid layer [7, 8]. In this talk, I will outline our recently developed experimental approaches to mitigate radiation damage and enable damage-free imaging of protein aggregate structure and other beam sensitive soft materials. These approaches include (1) the addition of radical scavengers [8] to selectively eliminate oxidizing radicals and (2) the use of advanced electron beam control during imaging to reduce the overall production of damaging radicals.

Protein aggregates of bovine serum albumin (BSA) were prepared either by heat-induced or agitationinduced denaturation and aggregation of a $30 \mathrm{mg} / \mathrm{mL}$ monomer solution. Protein aggregates had sizes on the order of $50-500 \mathrm{~nm}$, determined by dynamic light scattering. LCEM sample chips coated with positively charged poly-L-lysine were used to capture protein aggregates through attractive electrostatic interactions. For these experiments, we utilized Protochips microwell chips for protein aggregate imaging, as they enable creating very thin $(\sim 100 \mathrm{~nm})$ liquid layer that enable high spatial resolution imaging (Figure 1a). In some cases, protein aggregates were positively stained with 0.1 wt $\%$ uranyl acetate stain to enhance contrast (Figure 1b). The aggregates were imaged in both bright field TEM and STEM imaging modes, keeping total dose exposures below $\approx 10 \frac{\text { electron }}{\AA^{2}}$. Solutions of unaggregated BSA protein were observed to aggregate with repeated imaging, likely due to oxidative protein damage (Figure 2). Imaging experiments utilizing radical scavengers commonly used to prevent radiation damage during $\mathrm{x}$-ray scattering of proteins, including glycerol and tris buffer, have shown enhanced radiation protection. We will show promising results taking advantage of radiolysis kinetics and advanced STEM beam control to reduce the number of damaging radicals formed [9]. 


\section{References:}

[1] A.S. Rosenberg, Aaps Journal, 8 (2006) p. E501.

[2] M. Bucciantini, E. Giannoni, F. Chiti, et al., Nature, 416 (2002) p. 507.

[3] F.M. Ross, Science, 350 (2015).

[4] L.M. DiMemmo, A.C. Varano, J. Haulenbeek, et al., Lab on a Chip, 17 (2017) p. 315.

[5] K.A. Taylor, R.M. Glaeser, Science, 186 (1974) 1036.

[6] R.M. Glaeser, K.A. Taylor, Journal of Microscopy-Oxford, 112 (1978) p. 127.

[7] D. Elgrabli, W. Dachraoui, C. Menard-Moyon, et al., Acs Nano, 9 (2015) p. 10113.

[8] T.J. Woehl, P. Abellan, Journal of Microscopy, 265 (2017) p. 135.

[9] We acknowledge funding from the University of Maryland Seed Grant Program and thank Madeline Dukes for assistance with developing sample preparation approaches.

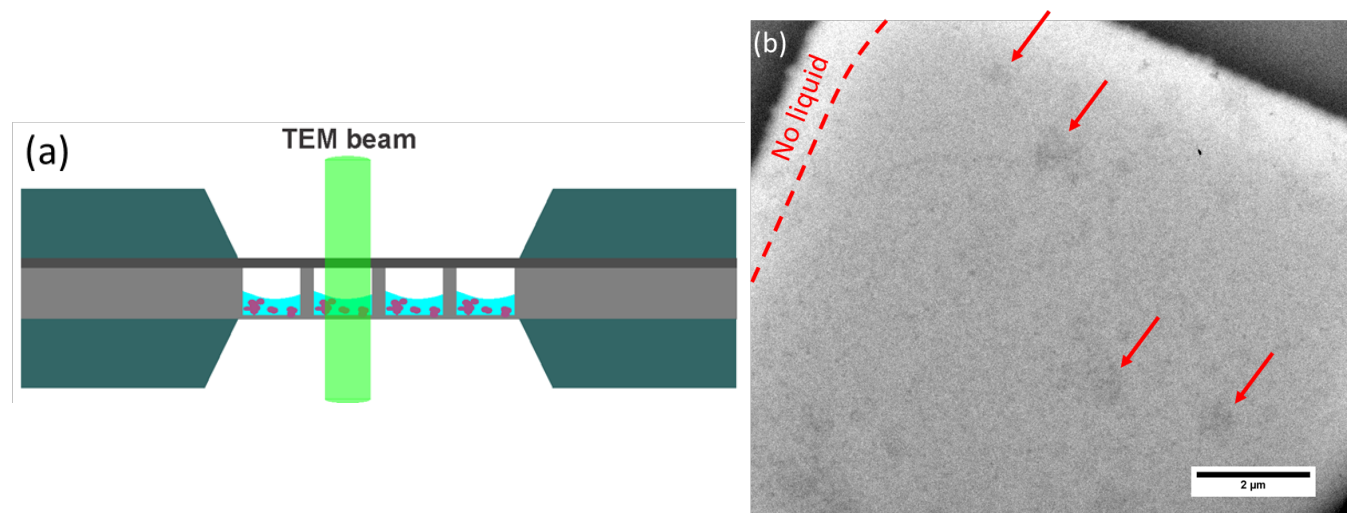

Figure 1. LCEM imaging of BSA aggregates formed by heat induced denaturation. Imaging was performed using microwell chips and bright field TEM on a $200 \mathrm{kV} \mathrm{LaB} 6$ TEM. (a) Schematic of protein aggregates in microwell chips. (b) Bright field TEM imaging of protein aggregates in a single microwell. The protein aggregates (red arrows) were positively stained with $0.1 \mathrm{wt} \%$ uranyl acetate. In this case the center of the microwell was hydrated, while the edges showed contrast consistent with the absence of liquid.
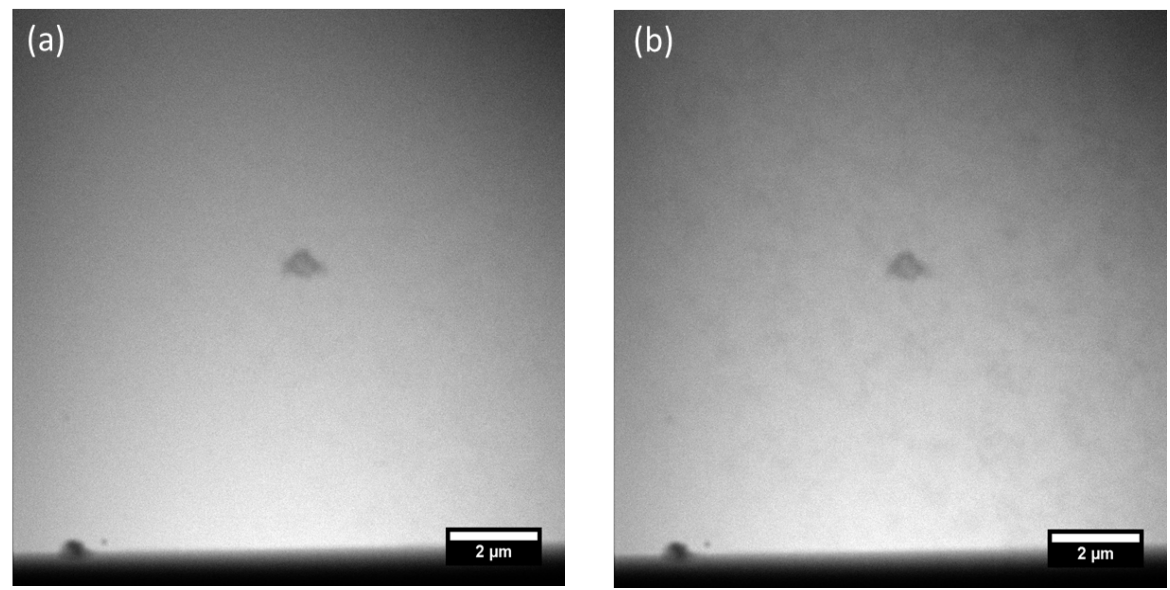

Figure 2. Electron beam induced aggregation of BSA during LCEM imaging with BF-STEM. (a) A solution of unaggregated BSA $(5 \mathrm{mg} / \mathrm{mL})$ in phosphate buffered saline after the $1^{\text {st }}$ (a) and $2^{\text {nd }}$ (b) STEM images were acquired. Each STEM image was acquired with a total dose of $\approx 10 \frac{\text { electrons }}{\AA^{2}}$. 